\title{
Added Value of Perfusion in Gynaecological Imaging
}

\author{
*Omneya Yakout Dogheim ${ }^{1}$ and Mohamed Eid ${ }^{2}$ \\ ${ }^{1}$ Assistant Lecturer, Alexandria Faculty of Medicine, Department of Diagnostic Imaging, Egypt \\ ${ }^{2}$ Assistant Professor, Alexandria Faculty of Medicine, Department of Diagnostic Imaging, Egypt
}

Submission: November 05, 2016; Published: November 23, 2016

"Corresponding author: Omneya Yakout Dogheim, Email: Omneyayakout@yahoo.com

\begin{abstract}
Owing to the high prevalence of gynecological malignancies worldwide, it is of utmost importance to detect these malignancies early, thus reducing their morbidity and mortality rates. Imaging plays a significant role not only in detection but also, owing to the new functional techniques including perfusion MRI, in staging and directing treatment planes. In this article we review the different perfusion techniques used in endometrial, cervical and ovarian malignancies and the importance and potential benefits for their use. The importance of early and proper diagnosis of gynecologic malignancies lies in the fact that they contribute to a large number of female mortality and morbidity worldwide.

Abbreviations: MRI: Magnetic Resonance Imaging; DCE-MRI: Dynamic Contrast Enhanced MRI; MVD: Microvessel Density; VEGF: Vascular Endothelial Growth Factor; IAUGC: Initial Area Under the Gadolinium Concentration-Time Curve; TAH-BSO: Total Abdominal Hysterectomy and Bilateral Salpingo-Oophorectomy; ESUR: European Society of Urogenital Radiology; FIGO: International Federation of Gynecology and Obstetrics; SI: Signal-Intensity Curve
\end{abstract}

\section{Introduction}

In 2006, 41,200 new uterine body cancer cases were diagnosed, 20,180 ovarian cancer cases, and 9,710 cervical cancer cases [1,2]. Diagnostic imaging plays an important role in diagnosing and staging gynecologic neoplasms and thus helps in treatment decision-making. Recent advances in MRI such as increased field strength, development of parallel imaging $[3,4]$, and the use of novel methods of rapid data acquisition have markedly improved image quality in body MRI applications [4]. MRI plays a pivotal role, owing to its good soft tissue resolution that allows accurate topographic assessment of the tumor (size, location, extension and nodal involvement) [5].

Unfortunately, conventional $\mathrm{T} 1$ and $\mathrm{T} 2$ sequences cannot provide adequate information about the tumor's microenvironment. Also, owing to the similar morphological appearance, conventional imaging has a limited value in assessing tumor's response to therapy and differentiating between residual /recurrent disease and post-treatment fibrosis [5,6]. Recently, the advancement of contrast enhanced Magnetic Resonance Imaging (MRI) has lead to offering an in vivo functional examination, where information on the structure and function of the tumor microvasculature can be provided [7]. Many studies now support the use of functional MRI in assessment of tumor response to therapy with promising results, particularly in cervical cancer [5]. DCE-MRI has the ability to noninvasively assess tumor perfusion and capillary permeability and thus assessing the anti angiogenic response of tumor tissue after therapy, which allows evaluation of treatment response earlier than using the conventional methods of assessing tumor size [8].

The early predictability of tumor response after therapy provides a golden opportunity to quickly change failed treatment regimens, limiting the related side effects and high costs and instituting these regimens with more successful ones $[5,9]$. Dynamic contrast enhanced MRI (DCE-MRI) is performed by acquisition of multiple sequential images before, during and after the passage of contrast material through a specific area of interest [8]. As the contrast material passes through a certain area, its paramagnetic effect increases the signal intensity; the degree of enhancement depends on various factors. In the early phase of enhancement (vascular phase), blood flow, vascular density, capillary permeability, and capillary surface area play a major role, while later in the interstitial phase, by extra vascular space volume [10].

This gives an idea about tumor microvascularity through variations in tissue enhancement over time. It has been shown that the more intense the enhancement of a tissue is, the more it is rich in vascularity. The rate of enhancement of a tissue (as demonstrated by the rate of wash-in and wash-out) is directly proportional to the expression of angiogenic factors such as micro vessel density (MVD) and vascular endothelial growth factor 


\section{Cancer Therapy \& Oncology International Journal}

(VEGF) [8]. So, variation in contrast enhancement is the result of variation in tumor histopathological features. Aggressive tumors mostly show rapid and intense enhancement and washout, due to high vascularity and strong expression of VEGF [8]. The use of post therapy DCE-MRI in cervical and endometrial cancer may be able to predict which patients are at risk of early recurrence and therefore super select patients suitable for more aggressive surgery [5]. In general, DCE perfusion MRI is very safe. Besides the general MRI risks and contraindications, IV infusion of a gadolinium- based contrast agent is done at a fairly rapid injection rate, so it is contraindicated in patients with renal failure [11].

\section{Ovarian cancer}

Five-year survival rates of patients diagnosed with ovarian cancer decreased from $90 \%$ in stage I to $30-40 \%$ in stage III and IV $[12,13]$. This dramatic decrease makes it of utmost importance to diagnose malignant ovarian masses as early as possible. DCE MRI is a non-invasive method that allows detection of ovarian malignancy early. Several studies were made proving its ability to differentiate between benign, borderline and high-grade ovarian tumors as well as its ability to differentiate between ovarian masses and pedunculated subserous uterine fibroid [7,14-16]. and to prove its ability to predict early response to therapy [10]. DCE MRI in cases of ovarian cancer is done using a T1-weighted gradient-echo sequence that is acquired so that the solid ovarian tumor tissue (i.e., solid portion, papillary projections, or thickened irregular septa) and the uterus are seen in a single image. After gadolinium injection, images are obtained at fivesecond intervals for two minutes, beginning immediately after the bolus injection. Followed by post contrast axial and sagittal T1- weighted gradient-echo images with breathhold [14,16].

Ovarian tumor detection and characterization: According to the enhancement pattern of the solid component within an adnexal mass, DCE MRI is able to differentiate between benign, borderline and malignant ovarian neoplasms. The more malignant lesions exhibit earlier and greater maximum enhancement. This variation of enhancement is attributed to the fact that malignant lesions have immature microvasculature with defective pericyte layer and over expression of VEGF receptors (VEGFR-2) $[7,15,16]$. DCE MRI can be performed by either qualitative or quantitative analyses.

In qualitative analysis, three curves are seen in the early phase of enhancement $(<2 \mathrm{~min})$, all are in comparison to the enhancement dynamics of the myometrium: $[7,15,16]$.

a) Type 1 curve: gradual enhancement never exceeding the enhancement of the myometrium, this is the benign curve (Figure 1).

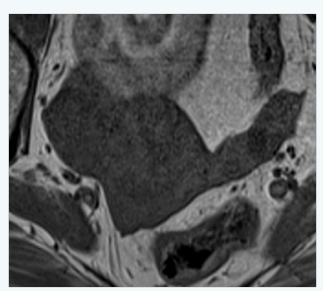

$1 \mathrm{a}$

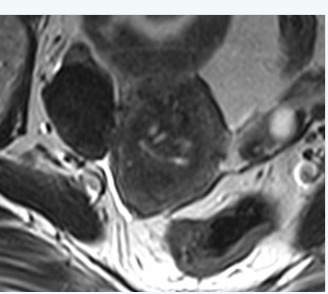

$1 \mathrm{~b}$

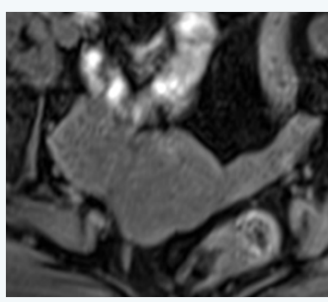

$1 \mathrm{c}$
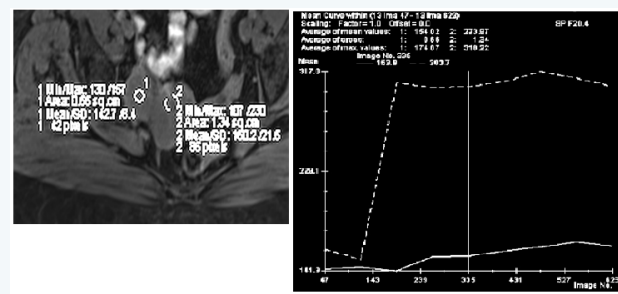

$1 \mathrm{~d}$

Figure 1: Ovarian Fibroma in a 61 years old patient

Figure 1a: T1W

Figure 1b: T2W MR images showing a well defined right sided solid hypointense adnexal mass

Figure 1c: Post-contrast T1W shows mild homogenus enhancement

Figure 1d: Dynamic contrast enhancement (DCE) curve shows a type 1 curve. The dotted line representing the enhancement of the myometrium and the continuous line is the enhancement of the solid mass

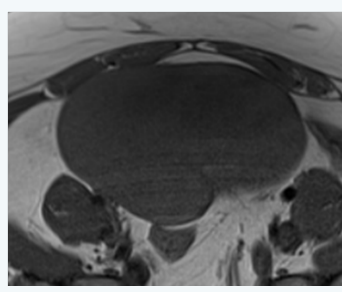

$2 a$

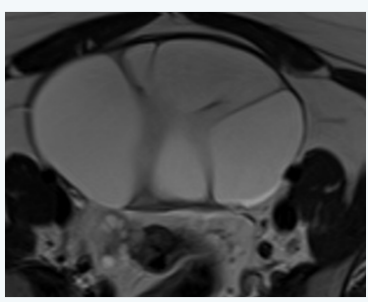

$2 b$

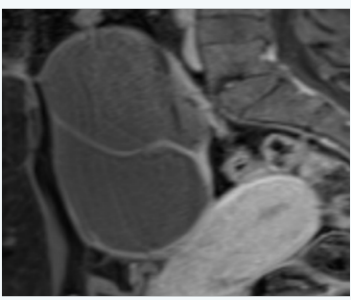

$2 c$

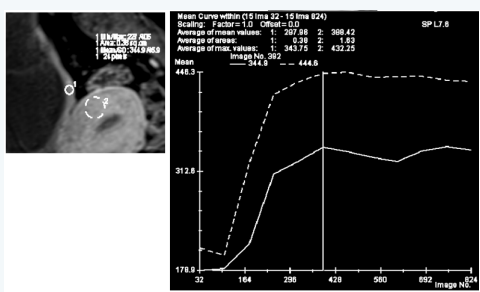

$2 d$

Figure 2: 37 years old patient with borderline mucinous cystadenocarcinoma

Figure 2a: Axial T1W

Figure 2b: Axial T2W MR images showing multi-cystic ovarian mass with homogeneous hypointense T1 signal and homogeneous hypointense T2 signal with multiple thick septations (>3 mm).

Figure 2c: sagittal postcontrast T1W images showing enhancement of these septations.

Figure 2d: DCE curve showing type 2 curve. 


\section{Cancer Therapy \& Oncology International Journal}

b) Type 2 curves: early enhancement exceeding that of the myometrium followed by a plateau (Figure 2). c) Type 3 curves: early and late enhancement exceeding that of the myometrium, this is specific to invasive malignancies (Figure 3).

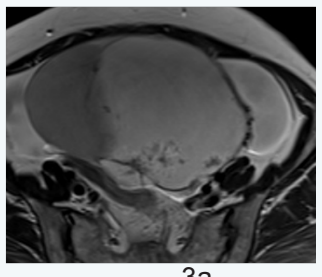

$3 a$

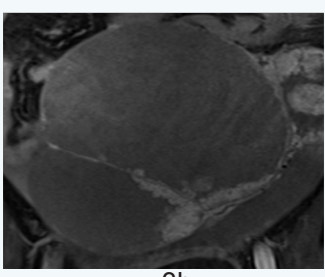

$3 \mathrm{~b}$

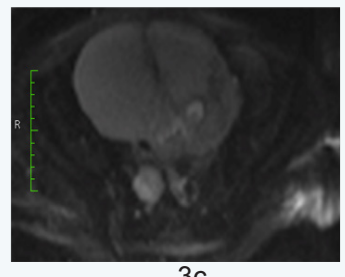

3c

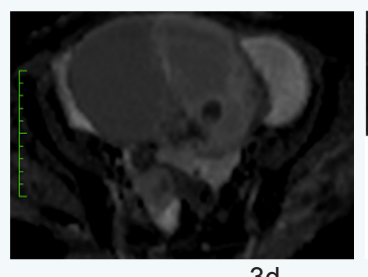

$3 d$

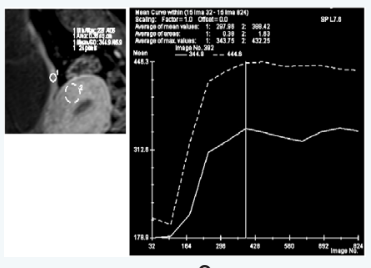

$3 e$

Figure 3: 33 years old women with invasive malignant cystadenocarcinoma

Figure 3a: Axial T2W showing stained glass appearance, suggestive of mucin containing lesion.

Figure 3b: Axial postcontrast T1W images, shows enhancing papillary projections.

Figure $3 c$ and $d: A D C$ and diffusion weighted images showing restricted diffusion in the solid projections.

Figure 3e: DCE curve is a type 3 curve ( malignant curve ). The continuous line represents the enhancement of the myometrium and the dotted line represents the enhancement of the solid components.

The importance of DCE MRI in ovarian cancer cases lies in its ability to differentiate between borderline and invasive malignancies. Since both are morphologically similar, so conventional MRI won't be of help. Patients with borderline ovarian tumors are young and usually want to preserve their fertility. And so, correct preoperative diagnosis and complete staging may give them the chance to do conservative surgeries $[14,17,18]$. Moreover, preoperative MRI suggesting borderline pathology was shown to help pathologists when intraoperative histology is required $[14,19]$.

Differentiating ovarian tumors from pedunculated subserous fibroids: Solid pelvic masses are sometimes very confusing, and when large, it is often impossible to determine their exact tissue of origin. Of special interest is the differentiation between ovarian fibromas and subserous uterine fibroids. Both are morphologically similar, however, it is important not to misdiagnose or confuse them owing to their different management options.

Both usually present as a T2-weighted solid homogenous or heterogeneous hypo intense mass with regular outlines [7]. DCE MRI easily differentiates between both, where ovarian fibromas show a type 1 curve (Benign curve) and subserous pedunculated fibroids show a type 2 curve (paralleling the myometrium) $[7,16]$ (Figure 4).

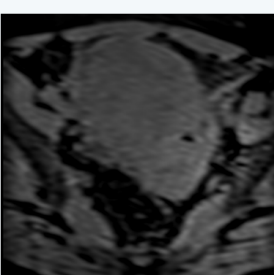

$4 a$

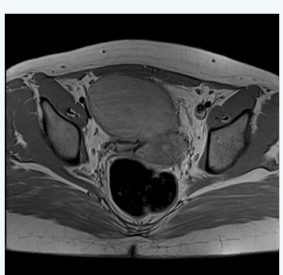

$4 b$

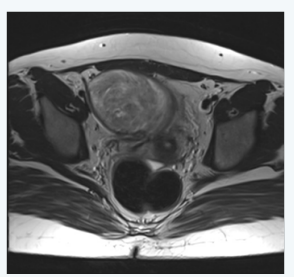

$4 c$

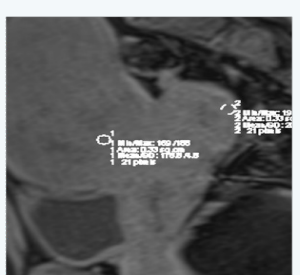

$4 d$

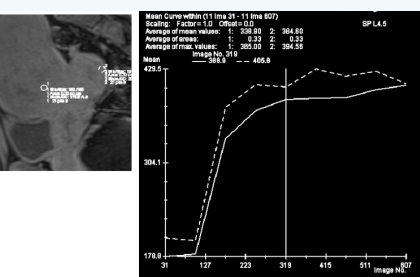

$4 d$

Figure 4: 20 years old women with a pelvic mass.

Figure 4a: Axial post-contrast T1W image showing a heterogeneously enhancing lesion.

Figure 4b and 4c: Axial T1 and T2W images showing isointense lesion with T2 hyperintensities.

Figure 4e: DCE curve shows a type 2 curve. This is a case of sub serous uterine fibroid. Ovarian fibroma would have similar MRI features but would show a type 1 curve.

The dotted curve represents the enhancement of the myometrium and the continuous line represents the enhancement of the solid mass.

Predicting response to treatment: Using quantitative DCE analysis,(within the region of interest),the following parameters can be calculated; the volume transfer constant (K trans), the rate constant (kep), the fractional volume of the extra vascular extracellular space (ve), the fractional plasma volume (vp) and the initial area under the gadolinium concentration-time curve
(IAUGC) [5,20]. Sala et al. [21,22] investigated the response of neo adjuvant chemotherapyin 22 patients with advanced primary ovarian cancer using both DWI and DCE-MR. In the primary ovarian lesion, responders showed a significantly larger increase in the ADC and in $\mathrm{V}(\mathrm{e})$ parameter of DCE as a result of the cytotoxic effect of platinum-based chemotherapy, but no 


\section{Cancer Therapy \& Oncology International Journal}

significant changes were found in omental or peritoneal deposits. The authors suggest the use of ADC and V (e) parameters as response markers to platinum-based therapy [22].

Predicting recurrent disease: In patients with asymptomatic residual disease after chemotherapy, Mitchell et al. [17] found that relapsed patients who eventually developed clinically progressive disease showed higher percent change of the enhancing fraction of the tumor 4-8 weeks post-baseline. Authors suggested that DCE MRI was able to predict relapse early. They also suggested that there is a positive relationship between the circulating tumor angiogenesis biomarkers (soluble vascular endothelial growth factor receptor-1 and -2) and the K trans, as well as a negative relationship between them and Vp $[10,22]$. This has the advantage of predicting chemo-resistance and therefore providing individualized patient treatment, reducing unnecessary costs and side effects [10].

\section{Endometrial Cancer}

Endometrial cancer is staged using the International Federation of Gynecology and Obstetrics (FIGO) system [23] Since the condition is predominantly treated surgically, staging according to the revised FIGO classification is mainly surgical [24]. Surgery is used to initially treat patients as well as to identify patients who need further therapy according to various factors, the most important of which is the depth of myometrial invasion, because it directly correlates with tumor grade, cervical involvement and the presence of lymph node metastasis [25-27]. The incidence of loco-regional lymph node metastases increases from $3 \%$ with superficial myometrial invasion (stage IA) to $46 \%$ with deep myometrial invasion (stage IB) [24,25].

Evaluation of the depth of myometrial invasion remains inaccurate in many cases by gross inspection at surgery or at frozen-section analysis [25,28,29]. The standard treatment of low-risk patients is total abdominal hysterectomy and bilateral salpingo-oophorectomy (TAH-BSO), with lymph node sampling of suspicious lymph nodes only. On the contrary, in high-risk patients, TAH-BSO as well as radical lymph node dissection should be made. This requires a specialized center with gynecologic-oncology surgeon [24]. Over-treating low risk patients results in increasing their morbidity, Furthermore, the recent introduction of laparoscopic techniques offers an alternative approach for these patients $[25,30,31]$

Accordingly preoperative MRI may play an important role owing to its ability to accurately detect the depth of myometrial invasion as well as the local and distant spread of endometrial tumors [24]. DCE MRI in cases of endometrial carcinoma is done using a 3DT1-weighted gradient- echo sequence. Images are taken before contrast medium injection and then at sagittal oblique planes at $1 \mathrm{~min}$, and $2 \mathrm{~min}$ after injection and ax axial oblique plane 3 min after injection [24]

\section{Endometrial Tumor Detection and Staging:}

A. Tumor detection: Endometrial cancers can be seen as a slowly enhancing area as compared to the enhancement of the surrounding myometrium [7]. So in the early phase of enhancement, a hypointense area is seen as compared to the myometrium, whereas later, an increase in the signal intensity is noted, which could be seen as a hypointense area (in $70 \%$ of patients), isointense area (in 20\%) or a hyperintense area (in $10 \%$ of cases) $[6,7]$. Unfortunately, there are no specific imaging findings to differentiate endometrial cancer from endometrial hyperplasia or endometrial polyps, so MRI plays a limited role in tumor characterization.

Some studies suggest that Diffusion weighted imaging and ADC value would be of help, where ADC value of carcinoma (0.88$0.98 \times 10-3 \mathrm{~mm} 2 / \mathrm{s}$ ) is significantly lower than of endometrial polyps (1.27-1.58 $\times 10-3 \mathrm{~mm} 2 / \mathrm{s})$ and of normal endometrium $(1.53 \times 10-3 \mathrm{~mm} 2 / \mathrm{s})$ [32]. To date, the standard of care is as follows; following presentation, the initial examination of choice is TVUS. Abnormally thick endometrium $(\leq 5 \mathrm{~mm}$ ) warrants endometrial sampling [33]. After histopathological confirmation of endometrial carcinoma, MRI is recommended for proper staging.

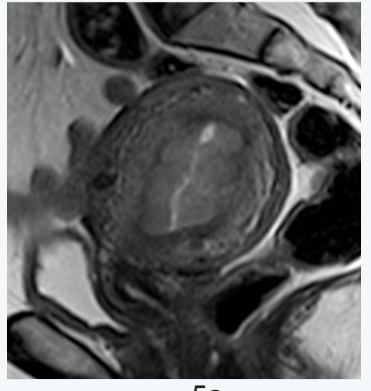

$5 a$

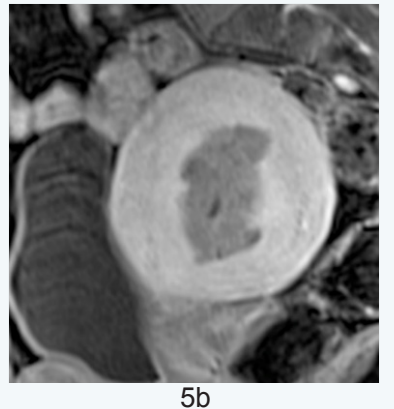

$5 b$
Figure 5: Known endometrial cancer presenting for staging

Figure 5a: sagittal T2W image showing endometrial thickening with suspicious involvement of the junctional zone.

Figure 5b: Dynamic T1W image clearly shows that the tumor involves less than $50 \%$ of the myometrium. This is stage $1 \mathrm{~A}$ cancer.

B. Tumor staging: DCE MRI plays a vital role in staging of endometrial cancer. Based on the FIGO system, it is important to determine the depth of the myometrial invasion and differentiate correctly between stages $1 \mathrm{~A} \quad(<50 \%$ myometrial invasion $)$ and stage $1 \mathrm{~B}(>50 \%)$. More than $50 \%$ myometrial invasion is associated with higher relapse rate, especially when combined with high tumor grade and histological subtype $(28,49)$. The incidence of presence of metastatic lymph nodes increases from $2.4 \%$ in low risk patients to $9 \%$ in intermediate risk patients and to $24 \%$ in high risks ones [24]. In order to adequately assess 


\section{Cancer Therapy \& Oncology International Journal}

the myometrial extension on conventional $\mathrm{T} 2 \mathrm{~W}$ images, the junctional zone has to be seen clearly. However, sometimes this is not the case. As hormones affect the junctional zone, it is not clearly visualized in post-menopausal women; in these cases assessment with combined DCE MRI and DWI is important [7] (Figures 5 \& 6).

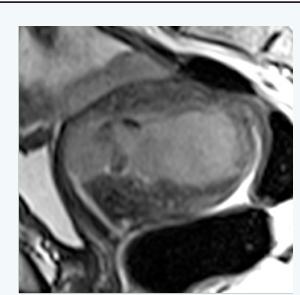

$6 a$

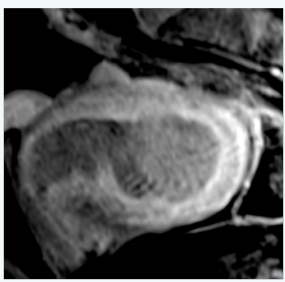

$6 b$

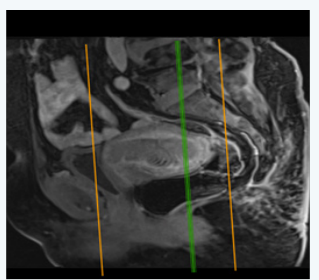

$6 c$

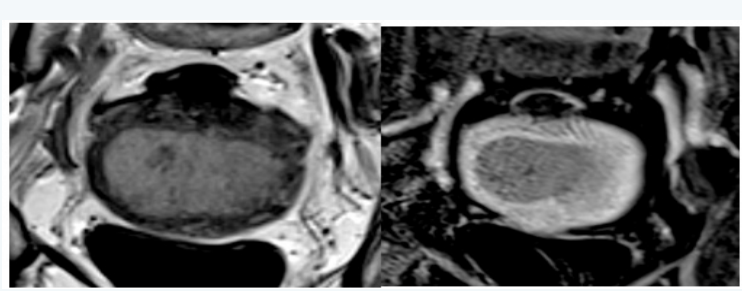

$6 d$

Figure 6: A patient with endometrial cancer presenting for staging.

Figure 6a: Sagittal T2W axial.

Figure 6b: Sagittal dynamic T1 postcontrast sequence (2 minutes after injection of contrast).

Figure 6c: Myometrium involvement was difficult to asses on the sagittal images. Oblique axial images on the uterine body are therefore important.

Figure 6d: Oblique axial T2W

Figure 6e: Oblique axial dynamic T1W after 3 minutes of contrast administration. Both showing $>50 \%$ myometrial invasion. This is a stage $1 \mathrm{~B}$ cancer.

It has been pointed out that at 50-120 seconds after contrast administration, the contrast between the hypointense endometrial tumor and the enhancing myometrium is maximum allowing for easy detection and accurate assessment of deep myometrial invasion. Delayed enhancement at 3-4 minutes is the best for assessing cervical stromal invasion (FIGO II) [24]. DCE MRI was shown to be useful if cervical extension is suspected because of the poorly vascularized cervical stroma [7].

C. Predicting response to treatment: The presence of hypoxia as a result of changes in tumor micovasculature and consequently changes in tumor perfusion one of the risk factors associated with resistance to treatment $[1,20]$. Theoretically speaking, the ability to measure the extent of hypoxia is of value in predicting response to therapy and to identify the patients who may benefit from additional therapeutic measures [5], but studies are lacking [10].

\section{Cervical cancer}

Staging of cervical cancer is also based on FIGO criteria, but in cervical cancer as opposed to endometrial cancer, the FIGO criteria are CLINICAL, owing to the fact that cervical cancer is most prevalent in developing countries with limited resources [24]. Clinical staging is somewhat misleading as compared to the surgical staging with nearly $32 \%$ of cases with stage IB disease and $65 \%$ with stage III disease being falsely staged. It is also highly inaccurate in predicting tumor size, parametrial invasion, pelvic sidewall invasion and lymph node metastasis, which are important prognostic factors that preoperative MRI excels at detecting $[24,25]$. It is important to distinguish early disease (with no parametrial invasion; stage I and IIA), which can be treated with surgery from advanced disease (with parametrial invasion; stage IIB and higher), which should be treated by chemo-radiotherapy [25]. DCE MRI in cervical carcinoma cases is not routinely done, as $\mathrm{T} 2 \mathrm{~W}$ images clearly detect the tumor as well as its extent [34]. DCE MRI in cervical carcinoma cases is done using a T1 turbo spin echo sequence in either the axial or coronal planes. Five acquisitions are obtained, each lasting for 30 seconds, starting after administering the whole dose of contrast material [6].

Cervical tumor detection: Cervical masses are seen as well defined $\mathrm{T} 2$ isointense areas within the $\mathrm{T} 2$ hypointense cervical fibrous stroma, which if small, can be difficult to visualize [7]. Several studies suggested that DCE MRI does not add additional information and its use is not encouraged [24,35]. However, other studies suggest that it could be helpful when the tumor is not visualized in T2W images, especially small tumors that should be considered for trachelectomy. The tumor enhances early (30 seconds) as compared to the cervical stroma, so early acquisitions will show the cervical mass as an enhancing area with surrounding non-enhancing stroma. However, late acquisitions will not be able to show the tumor as both, the tumor and the cervical stroma are enhancing [6-8].

Accordingly, DCE MRI should not be part of the routine MRI protocol of cervical carcinoma, and its use should only be limited to specific occasions; small tumors not seen on $\mathrm{T} 2 \mathrm{~W}$ images and in post-treatment follow up [47], as recommended by the European Society of Urogenital Radiology (ESUR) guidelines for staging cervical carcinomas $[24,34,36]$. Other studies suggest that DCE MRI also has a role is determining the exact endocervical extent of the tumor as well as differentiating between cervical and endometrial carcinomas [34]. 


\section{Cancer Therapy \& Oncology International Journal}

It is not advised to measure the tumor on DCE MRI as the enhancement of the surrounding normal stroma tends to exaggerate measurements [7].

Predicting Response to treatment and predicting recurrence: Internal areas of necrosis that are commonly seen in large tumors seen as low enhancing areas, an indicator of poor vascularity and hypoxia, are associated with poor response to treatment. On the other hand, highly vascular tumors are more radiosensitive [5], with lower rate of recurrence [10,37] (Figure 7). So DCE MRI has the theoretical advantage of noninvasively measuring tissue hypoxia, instead of using the invasive polarographic electrode. It is believed that tumors that respond well to early treatment show an increase in tumoral enhancement and have a lower rate of recurrence [10].

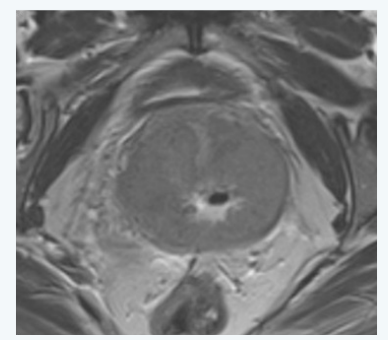

$7 a$

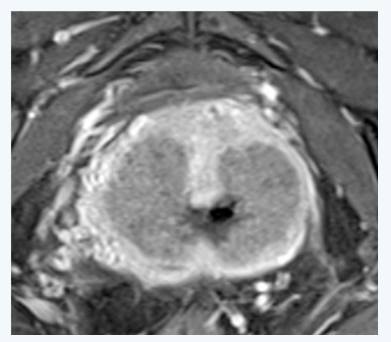

$7 \mathrm{~b}$
Figure 7: Cervical carcinoma.

Figure 7a: T2W MR images showing homogenous isointense cervical mass.

Figure 7b: Post-contrast T1W MR images showing extensive necrosis, a predictor of tumor hypoxia and poor response to treatment.

Accordingly, tumors with persistent low perfusion throughout treatment means poor treatment response and hence poor prognosis $[5,38,39]$. Low perfusion can be measured by:

i. Quantitative analysis, where some studies postulated low perfusion is the lower 10th percentile of the signalintensity curve (SI 10\%) [5,39], while other considered SI $15 \%$ is more specific $[5,40]$.

ii. Qualitative analysis, where low perfusion is less than that of the normal cervical tissue [5]. Still, DCE MRI is still used as an investigational method and its evaluation in this area is still ongoing $[6,10]$.

Post-therapy DCE-MRI to differentiate post-treatment fibrosis from recurrence: DCE MRI is able to differentiate between post-radiation fibrosis and early recurrence according to various studies [7]. It is believed that if persistent enhancement is present at the site of the cervical tumor or the surgical bed, residual disease is likely with high risk of recurrence and poor prognosis and adjunctive therapy should be advised [5,9,41]. In a small study conducted by Boss et al. [5,42] done on patients after radiotherapy, early enhancement at the original site of the tumor ( $<6$ seconds) was associated with poor prognosis and denoted recurrence, whereas delayed enhancement $(>6$ seconds) was associated with good prognosis and was the result of post-radiotherapy fibrosis. Still, these are all investigational studies and more studies are needed in this field.

\section{Conclusion}

DCE MRI is a valuable problem-solving tool in gynecological malignancies, which can answer a lot of questions guiding gynecologists to less invasive therapeutic options, which could preserve fertility in young women and decrease morbidity and mortality rates in the elder group.

\section{References}

1. Cancer facts and figures (2006) Ga: American Cancer Society, Atlanta, USA.

2. Viswanathan AN, Buttin BM, Kennedy AM (2008) Oncodiagnosis Panel: 2006. Ovarian, cervical, and endometrial cancer. Radiographics 28(1): 289-307.

3. Deshmane A, Gulani V, Griswold MA, Seiberlich N (2012) Parallel MR imaging. J Magn Reson Imaging 36(1): 55-72.

4. Nougaret S, Tirumani SH, Addley H, Pandey H, Sala E, et al. (2013) Pearls and pitfalls in MRI of gynecologic malignancy with diffusionweighted technique. AJR Am J Roentgenol 200(2): 261-276.

5. Hameeduddin A, Sahdev A (2015) Diffusion-weighted imaging and dynamic contrast-enhanced MRI in assessing response and recurrent disease in gynaecological malignancies. Cancer Imaging 15: 3.

6. Yamashita Y, Baba T, Baba Y, Nishimura R, Ikeda S, et al. (2000) Dynamic contrast-enhanced MR imaging of uterine cervical cancer: pharmacokinetic analysis with histopathologic correlation and its importance in predicting the outcome of radiation therapy. Radiology 216(3): 803-809.

7. Thomassin-Naggara I, Siles P, Balvay D, Cuenod CA, Carette MF, et al. (2013) MR perfusion for pelvic female imaging. Diagn Interv Imaging 94(12): 1291-1298.

8. Alvarez Moreno E, Jimenez de la Peña M, Cano Alonso R (2012) Role of New Functional MRI Techniques in the Diagnosis, Staging, and Follow up of Gynecological Cancer: Comparison with PET-CT. Radiol Res Pract 2012: 219546.

9. Harry VN (2010) Novel imaging techniques as response biomarkers in cervical cancer. Gynecol Oncol 116(2): 253-261.

10. Wakefield JC, Downey K, Kyriazi S, deSouza NM (2013) New MR techniques in gynecologic cancer. AJR Am J Roentgenol 200(2): 249260.

11. Essig M, Shiroishi MS, Nguyen TB, Saake M, Provenzale JM, et al. (2013) Perfusion MRI: the five most frequently asked technical questions. AJR Am J Roentgenol 200(1): 24-34.

12. Munkarah A, Chatterjee M, Tainsky MA (2007) Update on ovarian cancer screening. Curr Opin Obstet Gynecol 19(1): 22-26.

13. Carter JS, Koopmeiners JS, Kuehn-Hajder JE, Metzger GJ, Lakkadi N, et al. (2013) Quantitative multi parametric MRI of ovarian cancer. J Magn Reson Imaging 38(6): 1501-1509.

14. Thomassin-Naggara I, Daraï E, Cuenod CA, Rouzier R, Callard P, et al. (2008) Dynamic contrast-enhanced magnetic resonance imaging: a useful tool for characterizing ovarian epithelial tumors. J Magn Reson Imaging 28(1): 111-120.

15. Thomassin-Naggara I, Bazot M, Daraï E, Callard P, Thomassin J, et al (2008) Epithelial ovarian tumors: value of dynamic contrast-enhanced MR imaging and correlation with tumor angiogenesis. Radiology 248(1): 148-159. 


\section{Cancer Therapy \& Oncology International Journal}

16. Dogheim OY, AbdelHamid AEM, Barakat MS, Eid M, El-Sayed SM (2014) Role of novel magnetic resonance imaging sequences in characterization of ovarian masses. Egyptian J Radiol Nuclear Med 45(1): 237-251.

17. Fauvet R, Boccara J, Dufournet C, David-Montefiore E, Poncelet C, et al. (2004) Restaging surgery for women with borderline ovarian tumors: results of a French multicenter study. Cancer 100(6): 1145-1151.

18. Darai E, Tulpin L, Prugnolle H, Cortez A, Dubernard G (2007) Laparoscopic restaging of borderline ovarian tumors. Surg Endosc 21(11): 2039-2043

19. Bazot M, Nassar-Slaba J, Thomassin-Naggara I, Cortez A, Uzan S, et al. (2006) MR imaging compared with intraoperative frozen-section examination for the diagnosis of adnexal tumors; correlation with fina histology. Eur Radiol 16(12): 2687-2699.

20. Priest AN, Gill AB, Kataoka M, McLean MA, Joubert I, et al. (2010) Dynamic contrast-enhanced MRI in ovarian cancer: Initial experience at 3 tesla in primary and metastatic disease. Magn Reson Med 63(4) 1044-1049.

21. Sala E, Kataoka MY, Priest AN, Gill AB, McLean MA, et al. (2012) Advanced ovarian cancer: multiparametric MR imaging demonstrates response- and metastasis-specific effects. Radiology 263(1): 149-159.

22. Rockall A, Munari A, Avril N (2012) New ways of assessing ovarian cancer response: metabolic imaging and beyond. Cancer Imaging 12: 310-314.

23. Peter Beddy, Ailbhe C O'Neill, Adam K Yamamoto, Helen C Addley, Caroline Reinhold (2012) FIGO staging system for endometrial cancer: added benefits of MR imaging. Radiographics 32(1): 241-254.

24. Freeman SJ, Aly AM, Kataoka MY, Addley HC, Reinhold C, et al. (2012) The revised FIGO staging system for uterine malignancies: implications for MR imaging. Radiographics 32(6): 1805-1827.

25. Sala E, Wakely S, Senior E, Lomas D (2007) MRI of malignant neoplasms of the uterine corpus and cervix. AJR Am J Roentgenol 188(6): 15771587.

26. Berman ML, Ballon SC, Lagasse LD, Watring WG (1980) Prognosis and treatment of endometrial cancer. Am J Obstet Gynecol 136(5): 679688.

27. Boronow RC, Morrow CP, Creasman WT, Disaia PJ, Silverberg SG, et al. (1984) Surgical staging in endometrial cancer: clinical-pathologic findings of a prospective study. Obstet Gynecol 63(6): 825-832.

28. Goff BA, Rice LW (1990) Assessment of depth of myometrial invasion in endometrial adenocarcinoma. Gynecol Oncol 38(1): 46-48.

29. Quinlivan JA, Petersen RW, Nicklin JL (2001) Accuracy of frozen section for the operative management of endometrial cancer. BJOG 108(8): 798-803.
30. Wong CK, Wong YH, Lo LS, Tai CM, Ng TK (2005) Laparoscopy compared with laparotomy for the surgical staging of endometrial carcinoma. J Obstet Gynaecol Res 31(4): 286-290.

31. Eltabbakh GH, Shamonki MI, Moody JM, Garafano LL (2001) Laparoscopy as the primary modality for the treatment of women with endometrial carcinoma. Cancer 91(2): 378-387.

32. Namimoto T, Awai K, Nakaura T, Yanaga Y, Hirai T, et al. (2009) Role of diffusion-weighted imaging in the diagnosis of gynecological diseases. Eur Radiol 19(3): 745-760.

33. Patel S, Liyanage SH, Sahdev A, Rockall AG, Reznek RH (2010) Imaging of endometrial and cervical cancer. Insights Imaging 1(5-6): 309-328.

34. Bourgioti C, Chatoupis K, Moulopoulos LA (2016) Current imaging strategies for the evaluation of uterine cervical cancer. World J Radiol 8(4): 342-354.

35. Postema S, Pattynama PM, van Rijswijk CS, Trimbos JB (1999) Cervical carcinoma: can dynamic contrast-enhanced MR imaging help predict tumor aggressiveness? Radiology 210(1): 217-220.

36. Balleyguier C, Sala E, Da Cunha T, Bergman A, Brkljacic B, et al. (2011) Staging of uterine cervical cancer with MRI: guidelines of the European Society of Urogenital Radiology. Eur Radiol 21(5): 1102-1110.

37. Nina A Mayr, William TC Yuh, Jeffrey C Arnholt, James C Ehrhardt, Joel I Sorosky, et al. (2000) Pixel analysis of MR perfusion imaging in predicting radiation therapy outcome in cervical cancer. J Magn Reson Imaging 12(6): 1027-1033.

38. Mayr NA, Yuh WT, Magnotta VA, Ehrhardt JC, Wheeler JA, et al. (1996) Tumor perfusion studies using fast magnetic resonance imaging technique in advanced cervical cancer: a new noninvasive predictive assay. Int J Radiat Oncol Biol Phys 36(3): 623-633.

39. Mayr NA, Wang JZ, Zhang D, Grecula JC, Lo SS, et al. (2010) Longitudinal changes in tumor perfusion pattern during the radiation therapy course and its clinical impact in cervical cancer. Int J Radiat Oncol Biol Phys 77(2): 502-508.

40. Yuh WT, Mayr NA, Jarjoura D, Wu D, Grecula JC, et al. (2009) Predicting control of primary tumor and survival by DCE MRI during early therapy in cervical cancer. Invest Radiol 44(6): 343-350.

41. Hawnaur JM, Zhu XP, Hutchinson CE (1998) Quantitative dynamic contrast enhanced MRI of recurrent pelvic masses in patients treated for cancer. Br J Radiol 71(851): 1136-1142.

42. Boss EA, Massuger LF, Pop LA, Verhoef LC, Huisman HJ, et al. (2001) Post-radiotherapy contrast enhancement changes in fast dynamic MRI of cervical carcinoma. J Magn Reson Imaging 13(4): 600-606.

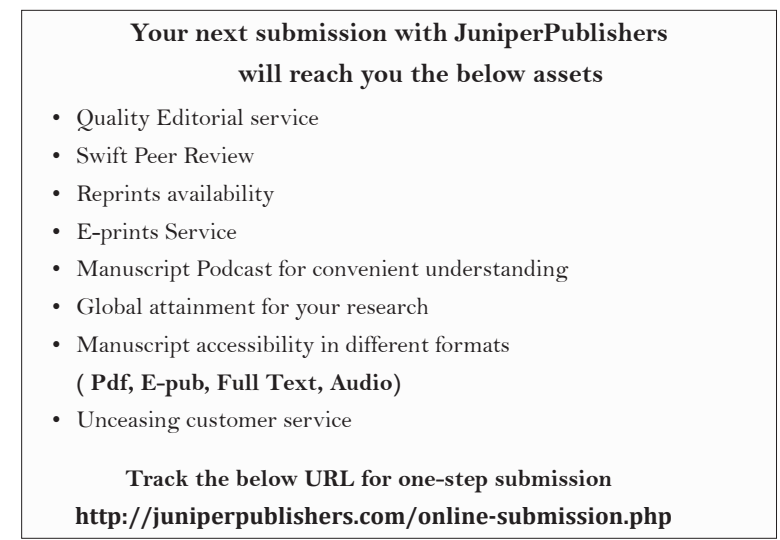

